\title{
Isolation of human monoclonal autoantibodies derived from pancreatic lymph node and peripheral blood B cells of islet autoantibody-positive patients
}

\author{
Mara Catani $^{1}$ • Denise Walther ${ }^{1}$ - Michael R. Christie ${ }^{2,3}$ - Kerry A. McLaughlin ${ }^{2}$. \\ Ezio Bonifacio $^{1,4}$ - Anne Eugster ${ }^{1}$
}

Received: 23 June 2015 / Accepted: 30 September 2015 / Published online: 22 October 2015

(C) Springer-Verlag Berlin Heidelberg 2015

\begin{abstract}
Aims/hypothesis Autoantibodies against pancreatic islets and infections by enteroviruses are associated with type 1 diabetes, but the specificity of immune responses within the type 1 diabetic pancreas is poorly characterised. We investigated whether pancreatic lymph nodes could provide a source of antigen-specific B cells for analysis of immune responses within the (pre)diabetic pancreas.

Methods Human IgG antibodies were cloned from single B lymphocytes sorted from pancreatic lymph node cells of three organ donors positive for islet autoantibodies, and from the peripheral blood of a patient with type 1 diabetes. Antibodies to insulinoma-associated antigen 2 (IA-2), GAD65, zinc transporter 8 (ZnT8) and Coxsackie B virus proteins were assayed by immunoprecipitation and by immunofluorescence on pancreatic sections.

Results Human IgG antibodies (863) were successfully cloned and produced from 4,092 single B cells from lymph
\end{abstract}

Electronic supplementary material The online version of this article (doi:10.1007/s00125-015-3792-4) contains peer-reviewed but unedited supplementary material, which is available to authorised users.

Anne Eugster

anne.eugster@crt-dresden.de

1 CRTD-DFG Research Center for Regenerative Therapies Dresden, Medical Faculty, Technische Universität Dresden, Fetscherstrasse 105, 01307 Dresden, Germany

2 Division of Diabetes and Nutritional Sciences, Guy's Campus, King's College London, London, UK

3 Present address: School of Life Sciences, University of Lincoln, Lincoln, UK

4 Paul Langerhans Institute Dresden of the Helmholtz Centre Munich at University Clinic Carl Gustav Carus, Technische Universität Dresden, Dresden, Germany nodes and peripheral blood. Reactivity to the protein tyrosine phosphatase domain of the IA-2 autoantigen was detected in two cloned antibodies: one derived from a pancreatic lymph node and one from peripheral blood. Epitopes for these two antibodies were similar to each other and to those for circulating antibodies in type 1 diabetes. The remaining 861 antibodies were negative for reactivity to IA-2, GAD65 or ZnT8 by both assays tested. Reactivity to a Coxsackie viral protein 2 was detected in one antibody derived from a peripheral blood B cell, but not from lymph nodes.

Conclusions/interpretation We show evidence for the infrequent presence of autoantigen-specific $\mathrm{IgG}^{+} \mathrm{B}$ lymphocytes in the pancreatic-draining lymph nodes of islet autoantibodypositive individuals.

Keywords Autoantibodies $\cdot$ Coxsackie virus $\cdot \operatorname{IgG}$ expression · Single-cell PCR · Type 1 diabetes mellitus

$\begin{array}{ll}\text { Abbreviations } \\ \text { BCR } & \text { B cell receptor } \\ \text { CDR3 } & \text { Complementarity determining region } 3 \\ \text { FLuc } & \text { Firefly luciferase } \\ \text { hAb } & \text { Human antibodies } \\ \text { HEK } & \text { Human embryonic kidney } \\ \text { HEV } & \text { Human enterovirus } \\ \text { IA-2 } & \text { Insulinoma-associated antigen } 2 \\ \text { JM } & \text { Juxtamembrane } \\ \text { LIPS } & \text { Luciferase immunoprecipitation system } \\ \text { PBMC } & \text { Peripheral blood mononuclear cells } \\ \text { PTP } & \text { Protein tyrosine phosphatase } \\ \text { RLuc } & \text { Renilla luciferase } \\ \text { VP } & \text { Viral protein } \\ \text { ZnT8 } & \text { Zinc transporter } 8\end{array}$




\section{Introduction}

Type 1 diabetes is associated with autoimmunity to islet cell proteins and with enteroviral infections in the inflamed pancreatic islet $[1,2]$. Little, however, is known about the specificity or site of immune responses during pancreatic inflammation in type 1 diabetes. Immune responses are expected to occur within the diabetic pancreas [3], but molecular mimicry or immunisation at sites distant to the islets has also been suggested [4]. Few studies have examined lymphoid cells within the pancreas for evidence of autoreactive $\mathrm{T}$ and $\mathrm{B}$ cells. Insulin-reactive $\mathrm{CD}^{+} \mathrm{T}$ cells have been demonstrated in pancreatic lymph nodes of a patient with long-standing type 1 diabetes [5], proinsulin-reactive $\mathrm{CD} 4^{+} \mathrm{T}$ cells have been cloned from pancreatic islets of a patient [6], and isletspecific glucose-6-phosphatase catalytic subunit-related protein-specific $\mathrm{CD}^{+} \mathrm{T}$ cells have been observed within islets in pancreas sections of a patient with type 1 diabetes [7]. B lymphocytes are often found within islets [8], but the specificity of those in and around the pancreas is unknown. We had the opportunity to study pancreatic lymph nodes of organ donors who were islet autoantibody-positive and investigated whether the B cells within these were specific for antigens implicated in the pathogenesis of type 1 diabetes.

\section{Methods}

\section{Donors and lymphocyte source}

Lymph nodes were obtained from pancreases of organ donors in the Nordic Network for Islet Transplantation (Table 1). Sera from organ donors were tested for autoantibodies to GAD65 and to insulinoma-associated antigen 2 (IA-2) and, if positive, pancreases were processed and lymphoid tissue retrieved. Multiple pancreatic lymph nodes were isolated from each of three islet autoantibody-positive organ donors (PLN1, PLN2 and PLN3). Lymph node cells were dissociated, washed and frozen in serum/DMSO. Peripheral blood mononuclear cells (PBMC) were obtained from a patient (PBMC1) with type 1 diabetes at onset of disease. Collection of material was approved by local ethics review boards (Regional Ethics Committee in Uppsala: Dnr 2009/043 and Dnr 2009/371 [2012 12 16]; Bayerische Landesärztekammer in Munich). CD19 $9^{+} \mathrm{B}$ lymphocytes were isolated by magnetic bead isolation (Miltenyi Biotec, Bergisch Gladbach, Germany), stained with anti-CD19-phycoerythrin and anti-IgG-FITC (BD Biosciences, San Diego, CA, USA), and single live $\mathrm{CD}_{1} 9^{+} \mathrm{IgG}^{+}$ lymphocytes sorted on a Becton Dickinson FACSAria II flow cytometer (Becton, Dickinson, Franklin Lakes, NJ, USA) into individual wells of PCR plates containing $10 \mu \mathrm{l}$ TRIS $+1 \mu \mathrm{l}$ RNasin (Promega, Madison, WI, USA).

\section{IgG production}

Single-cell RT and a first PCR with constant region 3' primers and a mix of 4-, 3- and 7-signal peptide sequence primers covering all subgroups of heavy chain, kappa and lambda light chains, respectively (electronic supplementary material [ESM] Table 1), was performed directly on cells. A second nested PCR was used to amplify the entire variable and partial constant regions of the chains, and to N-terminally fuse these to the METDTLLLVLLLWVPGSTGD signal peptide. This was assembled into the remaining constant region, with cytomegalovirus promoter and polyadenylation signal, and transfected into human embryonic kidney (HEK) 293 T cells using PolyFect Reagent (Qiagen, Hilden, Germany). After $48 \mathrm{~h}, \mathrm{IgG}$ concentration in supernatant fractions was measured by ELISA (ESM Fig. 1 and ESM Methods).

\section{Antibody screening}

Antigens of interest were N-terminally fused to Firefly luciferase (FLuc), Renilla luciferase (RLuc) or NanoLuc luciferase (Promega) and cloned into the pTNT vector (Promega). The following constructs were used to cover all known autoantibody epitopes of the antigens pTNTFLuc-IA-2 (amino acids 605-979), pTNTRLucGAD65 (96-585), pTNTFLuc-IA-2/zinc transporter 8 [ZnT8] WR (IA-2: 605-979; ZnT8W: 268-369, R325W; ZnT8R: 268-369), pTNTFLucZnT8W (268369, R325W) and pTNTRLucZnT8R (268-369), as well as human enterovirus (HEV) fusion constructs as described (ESM Table 2). Fusion proteins were produced using SP6- or T7-based TNT-coupled rabbit reticulocyte system (Promega). For screening of antibody specificity, $10 \mu \mathrm{l}$ clone supernatant fraction was incubated with $25 \mu \mathrm{l}$ luciferase fusion protein $\left(10^{7}\right.$ relative light units $[R L U] /$ well for FLuc or RLuc fusion proteins, $5 \times 10^{6}$ RLU/well for NanoLuc and for HEV fusion proteins) in TRIS-buffered saline/Tween 20 and immuneprecipitation assays performed (ESM Methods).

IA-2 epitope mapping of the human antibodies (hAb) was performed using constructs containing cytoplasmic IA-2 (amino acid 605-979), juxtamembrane (JM; 605693), protein tyrosine phosphatase (PTP; 643-979) or central IA-2 PTP (643-937) regions, and alaninesubstituted mutants of these, using radiobinding assays as previously described [9]. Inhibition by $>50 \%$ was taken as evidence that amino acid substitution affected binding.

Immunofluorescence (ESM Methods) was performed on ready-to-use frozen monkey pancreas sections (Bio-Diagnostics, Upton-upon-Severn, UK). 
Table 1 Cells and monoclonal antibodies produced and analysed, with description of samples used and characteristics of type 1 diabetic patients

\begin{tabular}{|c|c|c|c|c|c|c|}
\hline B lymphocyte source & $\begin{array}{l}\text { GAD65/IA-2 } \\
\text { antibodies, U/ml }\end{array}$ & $\begin{array}{l}\mathrm{HbA}_{1 \mathrm{c}}, \% \\
(\mathrm{mmol} / \mathrm{mol})\end{array}$ & HLA & $\begin{array}{l}\mathrm{IgG}^{+} \mathrm{CD} 19^{+} \\
\text {cells isolated, } n\end{array}$ & $\begin{array}{l}\text { Successful heavy and } \\
\text { light chain products, } \\
n(\%)\end{array}$ & $\begin{array}{l}\text { Productive } \\
\text { hAb, } n(\%)\end{array}$ \\
\hline $\begin{array}{l}\text { PLN1 } \\
\text { Female islet donor } \\
\text { Age at sampling: } 37 \text { years } \\
\text { Pancreatic lymph nodes }\end{array}$ & $10 / 30$ & $5.3(34.4)$ & $\mathrm{A} 3 / 31, \mathrm{~B} 35 / 39, \mathrm{DR} 1 / 9$ & 576 & $335(58)$ & $215(37)$ \\
\hline $\begin{array}{l}\text { PLN2 } \\
\text { Male islet donor } \\
\text { Age at sampling: } 63 \text { years } \\
\text { Pancreatic lymph nodes }\end{array}$ & $13 / 64$ & $5.2(33.3)$ & $\mathrm{A} 1 / 30, \mathrm{~B} 18 / 60, \mathrm{DR} 1 / 3$ & 768 & $196(26)$ & $105(14)$ \\
\hline $\begin{array}{l}\text { PLN3 } \\
\text { Male islet donor } \\
\text { Age at sampling: } 59 \text { years } \\
\text { Pancreatic lymph nodes }\end{array}$ & $10,600 /$ negative & $5.8(39.9)$ & $\mathrm{A} 3 / 10, \mathrm{~B} 7 / 12, \mathrm{DR} 3 / 15$ & 1,488 & $459(31)$ & $261(17.5)$ \\
\hline $\begin{array}{l}\text { PBMC1 } \\
\text { Male donor with new-onset } \\
\text { type } 1 \text { diabetes } \\
\text { Age at sampling: } 30.5 \text { years } \\
\text { (0.2 years after onset) } \\
\text { PBMC }\end{array}$ & $1,000 / 1,000$ & NA & $\begin{array}{l}\text { HLA DRB } 1 * 03 / * 04 \\
\quad \text { DQB } 1 * 02 / * 0302\end{array}$ & 1,260 & $536(43)$ & $282(22)$ \\
\hline Total & & & & 4,092 & $1,526(37)$ & $863(21)$ \\
\hline
\end{tabular}

NA, not available

\section{Results}

\section{Generation of hAb from single $\mathrm{CD}^{+} 9^{+} \mathrm{IgG}^{+} \mathrm{B}$ lymphocytes}

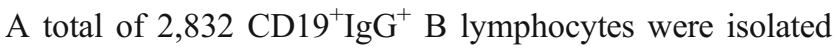
from pancreatic lymph nodes and 1,260 from PBMC (Table 1 ). IgG heavy and light chain variable region pairs were amplified from 1,526 of 4,092 single cells (37\% PCR efficiency, comparable to data given in [10]), and, after heavy and light chain assembly and transfection of HEK 293 T cells, IgG was detected in 863 (21\% of isolated B cells) of the supernatant fractions.

Complementarity determining region 3 (CDR3) of the heavy and light chain PCR products from PLN1 and from PBMC1 were sequenced, and 571 heavy chain, 280 kappa light chain and 125 lambda light chain readable sequences were obtained (www.crt-dresden.de/research/crtd-coregroups/bonifacio.html, accessed 1 June 2015; ESM Fig. 2). The same heavy or light chain sequence was not found in more than one cell from lymph nodes, but examples were seen in cells derived from peripheral blood (not shown).

\section{Identification of islet autoantigen-specific hAb}

The hAb were tested for their ability to bind the major autoantigens GAD65 and IA-2 and the two isoforms of ZnT8 ( $\mathrm{R}$ and $\mathrm{W})$. Four hAb showed increased binding to the IA-2/ZnT8R/W fusion protein (Fig. 1a), and two of these (PBMC1_A5 and PLN1_C11) were subsequently shown to strongly bind IA-2-luciferase, but not ZnT8R- or ZnT8Wluciferase (Fig. 1b). No hAb bound GAD65-luciferase.

Both PBMC1_A5 and PLN1_C11 bound the PTP- and the central region constructs, indicating that their epitopes are included within amino acids 643-937 of IA-2 (Fig. 1c). Reactivity was further characterised using single amino acid mutants within the region $826-862$, previously shown to encompass a major IA-2 epitope [9] (Fig. 1d). The binding profiles for the two hAb were almost identical and similar to that of existing hAb 96/3, despite different CDR3 amino acid sequences (PBMC1_A5: heavy chain CAKDSHVVAATPFDYW, light chain CQTWATGL\#VF; PLN1_C11: heavy chain CAKEKWNFYDGSGYSSGYDYW, light chain CQQAKDFPITF; 96/3: heavy chain CARVLKGVATASFDFW, light chain CQVWDTRSDLVVF; ESM Table 3). Binding was markedly reduced by the mutations L831A, V834A, E836A, L839A, K857A, N858A, V859A or Q862A.

$\mathrm{All} \mathrm{hAb}$ were also tested for reactivity to pancreatic islets, using immunofluorescence on sections of monkey pancreas. A strong islet-specific signal could be detected with the IA-2binding PBMC1_A5 and with the control IA-2-specific hAb 96/3, but not with the IA-2-binding PLN1_C11 (Fig. 1e). None of the remaining hAb showed reactivity to monkey pancreas sections.

\section{Reactivity of the hAb against HEV antigen}

Type 1 diabetes is associated with Coxsackie B virus infection, with evidence of enteroviral proteins present in the inflamed islet [2]. We therefore examined whether any of the $863 \mathrm{hAb}$ bound 
a

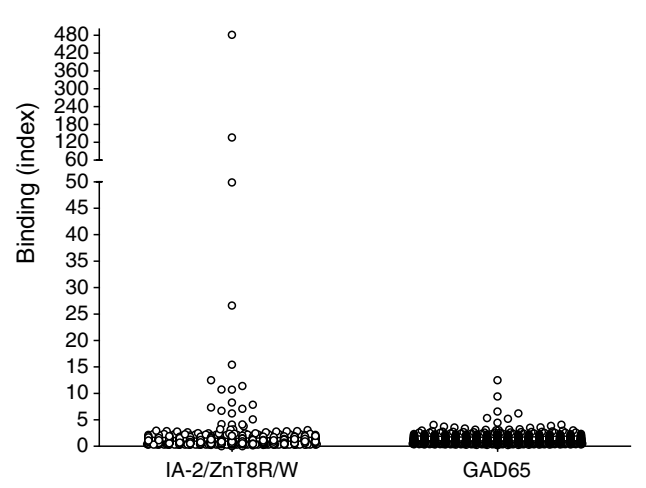

b

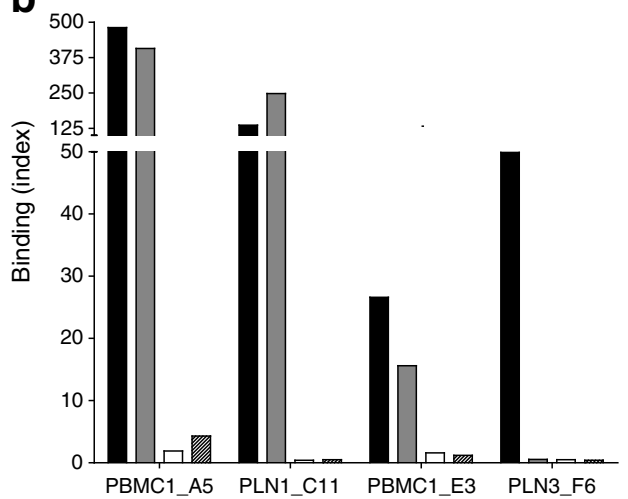

C

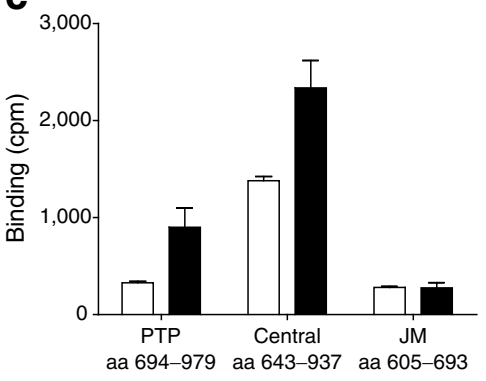

d

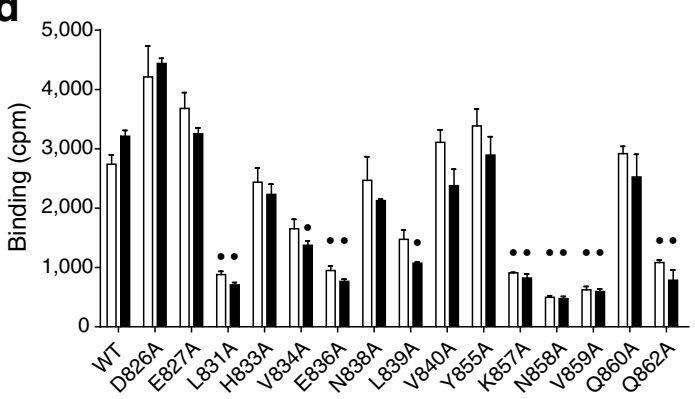

e

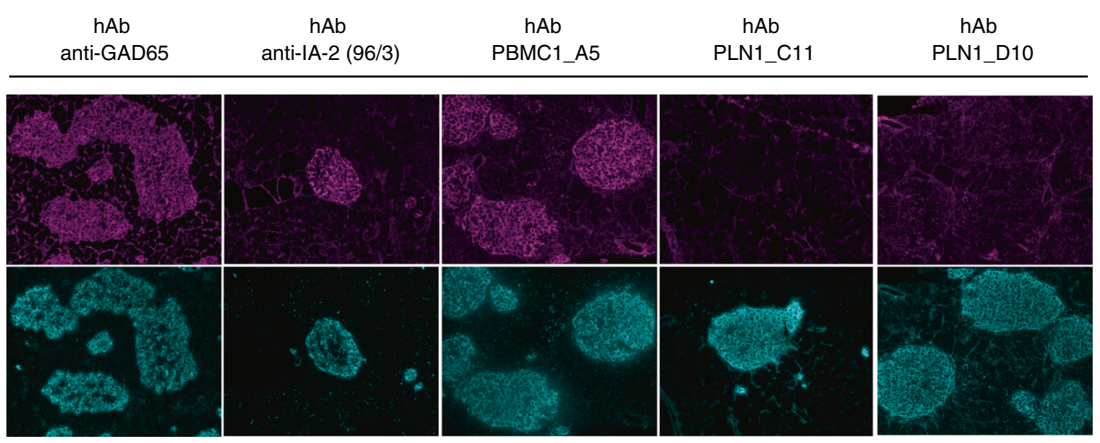

mAb anti-insulin

Fig. 1 (a) Immunoprecipitation assays for reactivity against IA-2, ZnT8 and GAD65. Dual-luciferase LIPS assays were performed on $863 \mathrm{hAb}$ with FLuc fused to the IA-2 and ZnT8R/W antigens and RLuc fused to the GAD65 antigen. Binding was expressed as an index against the negative control. (b) Four hAb bound to the IA-2/ZnT8R/W fusion protein (PBMC1_A5, PLN1_C11, PBMC1_E3 and PLN3_F6) with an index $>20$, and were tested using single constructs containing IA-2, ZnT8R and ZnT8W. PBMC1_A5 and PLN1_C11 showed reactivity only for IA-2. PBMC1_E3 and PLN3 F6 did not bind any of these fusion proteins (index $<20$ ). None of the $\mathrm{hAb}$ were considered positive against GAD65 (a). Black bars, IA-2/ZnT8R/W; grey bars, IA-2; white bars, ZnT8R; striped bars, ZnT8W. (c) Mapping of the binding region of PBMC1_A5 (white bars) and PLN1_C11 (black bars). Using epitope mapping with

constructs representing the majority of the viral protein (VP) antigens found in Coxsackie B virus 1-6 using luciferase immunoprecipitation system (LIPS) assays (ESM Fig. 3). Only one hAb, PBMC1_A6, bound Coxsackie B virus protein, specifically the luciferase-CV-B1 VP2. Serum obtained from the same patient was positive against the same construct (not shown).
IA-2 deletion mutants (indicated on the $x$-axis), both hAb were shown to react with the IA-2 region 643-979 (PTP and central regions) in radiobinding assays. Binding is expressed as counts per minute (cpm). (d) Fine-mapping of the binding region of PBMC1_A5 and PLN1_C11. Single mutations in the central PTP region (aa 826 to aa 862 , indicated in the $x$-axis) revealed that PBMC1 A5 and PLN1 C11 had comparable IA-2 amino acid requirements for binding. "Antibody binding $<50 \%$ with respect to the wild-type protein. (e) Staining of monkey pancreatic sections with $\mathrm{hAb}$ (upper panels, magenta); staining with PBMC1 A5 and PLN1_C11, with anti-IA-2 (96/3) and GAD65 hAb as positive controls and a GAD-, IA-2- and ZnT8-negative antibody (PLN1_D10) as negative control, and co-staining for insulin (lower panels, cyan). aa, amino acid; $\mathrm{mAb}$, monoclonal antibody

\section{Discussion}

We sought evidence for B cells against autoantigen and Coxsackie $\mathrm{B}$ virus in the lymph nodes of islet autoantibodypositive organ donors. One pancreatic lymph node-derived B cell was specific for IA-2 and therefore provided evidence for 
$\mathrm{B}$ cell priming against autoantigen, but we found no evidence of $B$ cells reactive to Coxsackie $B$ virus in the pancreatic lymph nodes.

We had expected pancreatic lymph node B cells to be an enriched source of islet autoantibodies. However, B cells directed against pancreatic islet antigens were rare in the lymph nodes we studied. A previous study examining plasma cells in the cerebrospinal fluid of patients with multiple sclerosis also showed low frequencies of autoantigen-specific antibody-producing $\mathrm{B}$ cells in the vicinity of the lesions [11]. Unfortunately we could not investigate binding to one of the major islet autoantigens, insulin, due to high interference in the assays. We cannot exclude that the low frequency of islet antigen reactive $\mathrm{B}$ cells detected in our study was the consequence of sequence artefacts introduced during the cloning that affected the CDR3-binding region of the B cell receptors (BCRs). Nevertheless, the study produced two antibodies against IA-2, one from a lymph node and one from peripheral blood, both to the PTP region, with binding profiles similar to each other and to other type 1 diabetes-derived autoantibodies [9]. This epitope, therefore, is a common target of autoreactive B cells in type 1 diabetes. Subtle differences in the fine specificity of the two IA-2 autoantibodies were shown on the basis of reactivity with mutated IA-2 constructs and heterogeneous reactivity with islets on monkey pancreas. Absent reactivity on monkey pancreas for one antibody may be due to an amino acid difference at position 862 between human and macaque IA-2.

Because of reports of HEV infection in the type 1 diabetic pancreas [2], we looked for evidence of pancreatic lymph node $\mathrm{B}$ cells directed to Coxsackie proteins. Although we were able to identify one Coxsackie B virus-specific BCR from peripheral blood B cells, we did not see binding from the lymph node-derived BCRs. This may either indicate that the patients had little or no exposure to Coxsackie B virus in the pancreas or that our methods were inadequate to faithfully produce $\mathrm{hAb}$.

In summary, we provide evidence that there are $\operatorname{IgG}^{+} \mathrm{B}$ lymphocytes primed against islet autoantigen at low frequency in lymph nodes that drain the pancreas of islet autoantibody-positive individuals.

Acknowledgements We thank S. Tuncel and R. Lutz (CRTD-DFG Research Center for Regenerative Therapies Dresden, Germany) for their technical assistance. We kindly thank V. Lampasona (San Raffaele Institute, Milan, Italy), the late J. Hutton (Barbara Davis Center for Childhood Diabetes, University of Colorado, Denver, CO, USA) and S.Gambhir (Stanford University, Stanford, CA, USA) for sharing plasmids and constructs, K. Klingel (Institute of Pathology and Neuropathology, University of Tübingen,
Tübingen, Germany) for sharing antibodies, and M. Roivainen (National Institute for Health and Welfare, Helsinki, Finland) for sharing reagents and providing viral sequences. Human tissues were obtained from the Nordic Network for Clinical Islet Transplantation, supported by: the Swedish national strategic research initiative Excellence of Diabetes Research in Sweden (EXODIAB); European Union Framework Programme 7 (EU-FP7): Diabetes Type 1 Prediction, Early Pathogenesis and Prevention (DIAPREPP); and the JDRF.

\section{Compliance with ethical standards}

Funding Funding was provided by the following research grants: JDRF 17-2012-593 (Targets of patient pancreatic lymph node $\mathrm{IgG}^{+}$B lymphocyte); EU-FP7 (DIAPREPP 202013); and Diabetes UK 11/0004297.

Duality of interest The authors declare no conflict of interest.

Contribution statement $\mathrm{EB}$ and $\mathrm{AE}$ contributed to the conduct of the study and the acquisition, analysis and interpretation of data, and drafted, reviewed and approved the manuscript. MC, DW, MRC and KAM contributed to the acquisition, analysis and interpretation of data, and drafted, reviewed and approved the manuscript. All authors approved the final version of the manuscript. $\mathrm{AE}$ and $\mathrm{EB}$ are the guarantors of this work.

\section{References}

1. Ziegler AG, Nepom GT (2010) Prediction and pathogenesis in type 1 diabetes. Immunity 32:468-478

2. Richardson SJ, Morgan NG, Foulis AK (2014) Pancreatic pathology in type 1 diabetes mellitus. Endocr Pathol 25:80-92

3. van Belle TL, Coppieters KT, von Herrath MG (2011) Type 1 diabetes: etiology, immunology, and therapeutic strategies. Physiol Rev 91:79-118

4. Afonso G, Mallone R (2013) Infectious triggers in type 1 diabetes: is there a case for epitope mimicry? Diabetes Obes Metab 15:82-88

5. Kent SC, Chen Y, Bregoli L et al (2005) Expanded T cells from pancreatic lymph nodes of type 1 diabetic subjects recognize an insulin epitope. Nature 435:224-228

6. Pathiraja V, Kuehlich JP, Campbell PD et al (2015) Proinsulin-specific, HLA-DQ8, and HLA-DQ8-transdimer-restricted CD4+ T cells infiltrate islets in type 1 diabetes. Diabetes 64:172-182

7. Coppieters KT, Dotta F, Amirian N et al (2012) Demonstration of islet-autoreactive CD8 T cells in insulitic lesions from recent onset and long-term type 1 diabetes patients. J Exp Med 209:51-60

8. Arif S, Leete P, Nguyen V et al (2014) Blood and islet phenotypes indicate immunological heterogeneity in type 1 diabetes. Diabetes 63:3835-3845

9. McLaughlin KA, Richardson CC, Williams S et al (2015) Relationships between major epitopes of the IA-2 autoantigen in type 1 diabetes: implications for determinant spreading. Clin Immunol 160:226-236

10. Murugan R, Imkeller K, Busse CE et al (2015) Direct highthroughput amplification and sequencing of immunoglobulin genes from single human B cells. Eur J Immunol 45:2698-2700

11. Owens GP, Bennett JL, Lassmann H et al (2009) Antibodies produced by clonally expanded plasma cells in multiple sclerosis cerebrospinal fluid. Ann Neurol 65:639-649 